\title{
ON A CHARACTERISTIC PROPERTY OF POINT PROCESSES
}

\author{
B.L.S. PRAKASA RAO
}

(Received 10 July 1974; revised 17 December 1974)

Communicated by C. C. Heyde

\section{Abstract}

This note is concerned with a certain property of point processes. We prove that if $N_{1}, N_{2}$ and $N_{3}$ are three independent point processes, then the bivariate point process $\left(N_{1}+N_{3}, N_{2}+N_{3}\right)$ uniquely determines the point processes $N_{1}, N_{2}$ and $N_{3}$.

\section{Main result}

It is known that every point process $N(\cdot)$ corresponds to a triple $\left(\Omega, \mathscr{F}, P_{N}\right)$ where $\Omega$ is the set of all countable sequences of real numbers $\left\{t_{i}\right\}$ without limit points and $\mathscr{F}$ is the $\sigma$-algebra generated by cylinder sets and $P_{N}$ is a probability measure (cf. Harris (1963)). We say that the point process $N(\cdot)$ is degenerate if $P_{N}$ is concentrated at a single point $\left(r_{1}, r_{2}, \cdots\right)$ in $\Omega$. Let $V$ denote the set of measurable functions $\xi$ such that $0 \leqq \xi(t) \leqq 1$ for all real $t$ and $\xi(t)=1$ outside a bounded interval.

DEFINITION 2.1. The probability generating functional (p.g.f) of a point process $N(\cdot)$ is defined by

$$
G(\xi)=E\left\{\exp \int \log \xi(t) d N(t)\right\}
$$

for $\xi \in V$. (If $\xi(t) \equiv 0$ over some set $A$ the exponential in (1) is taken as zero unless $N(A)=0$ when it equals one $)$.

DEFINITION 2.2. The p.g.fl of a bivariate point process $\left(M_{1}(\cdot), M_{2}(\cdot)\right)$ is defined by 


$$
H\left(\xi_{1}, \xi_{2}\right)=E\left\{\exp \left[\int \log \xi_{1}(t) d M_{1}(t)+\int \log \xi_{2}(t) d M_{2}(t)\right]\right\}
$$

for $\xi_{1} \in V, \xi_{2} \in V$.

THËOREM 2.1. Let $N_{1}, N_{3}$ and $N_{2}$ be three independent point processes and let $M_{1}=N_{1}+N_{3}$ and $M_{2}=N_{3}+N_{2}$. Then the bivariate point process $\left(M_{1}, M_{2}\right)$ uniquely determines the point processes $N_{1}, N_{3}$ and $N_{2}$.

Proof. Let $G_{1}(\xi), G_{3}(\xi), G_{2}(\xi)$ and $H\left(\xi_{1}, \xi_{2}\right)$ denote the p.g.fl's of $N_{1}, N_{3}$, $N_{2}$ and $\left(M_{1}, M_{2}\right)$ respectively. It is easy to see that

$$
\begin{aligned}
H\left(\xi_{1}, \xi_{2}\right)= & E\left\{\exp \left[\int \log \xi_{1}(t) d M_{1}(t)+\int \log \xi_{2}(t) d M_{2}(t)\right]\right\} \\
= & E\left\{\operatorname { e x p } \left[\int \log \xi_{1}(t) d N_{1}(t)+\int \log \xi_{2}(t) d N_{2}(t)\right.\right. \\
& \left.\left.+\int \log \left(\xi_{1}(t) \xi_{2}(t)\right) d N_{3}(t)\right]\right\} \\
= & G_{1}\left(\xi_{1}\right) G_{2}\left(\xi_{2}\right) G_{3}\left(\xi_{1} \xi_{2}\right)
\end{aligned}
$$

for $\xi_{1} \in V, \xi_{2} \in V$ since $N_{1}, N_{2}$ and $N_{3}$ are independent point processes. Suppose now that $R_{1}, R_{3}, R_{2}$ are independent point processes such that the bivariate point process $\left(S_{1}, S_{2}\right)$ has the same probability structure as $\left(M_{1}, M_{2}\right)$ where $S_{1}=R_{1}+R_{3}$ and $S_{2}=R_{3}+R_{2}$. Let $K_{1}(\xi), K_{3}(\xi)$ and $K_{2}(\xi)$ be the p.g.fl's of $R_{1}, R_{3}$ and $R_{2}$ respectively. It is now easy to see that

$$
H\left(\xi_{1}, \xi_{2}\right)=K_{1}\left(\xi_{1}\right) K_{2}\left(\xi_{2}\right) K_{3}\left(\xi_{1} \xi_{2}\right)
$$

Let $A_{j}, 1 \leqq j \leqq K$ be disjoint Borel sets in $R^{1}$ and let $G_{i}(z)$ and $K_{i}(z)$ denote the p.g.fl's of $\left(N_{i}\left(A_{1}\right), \cdots, N_{i}\left(A_{K}\right)\right)$ and $\left(R_{i}\left(A_{1}\right), \cdots, R_{i}\left(A_{K}\right)\right)$ respectively. Then (3) and (4) imply that

$$
G_{1}\left(z_{1}\right) G_{2}\left(z_{2}\right) G_{3}\left(z_{1} z_{2}\right)=K_{1}\left(z_{1}\right) K_{2}\left(z_{2}\right) K_{3}\left(z_{1} z_{2}\right)
$$

for all $z \in[0,1]^{K}$ where $z_{1} z_{2}$ is the vector obtained by multiplying $z_{1}$ and $z_{2}$ coordinate wise. $G_{i}(z)$ and $K_{i}(z), 1 \leqq i \leqq 3$ are non zero in the set $D^{*}=$ $\left\{0<z_{j} \leqq 1,1 \leqq j \leqq K\right\}$ where $z=\left(z_{1}, \cdots, z_{K}\right)$. Let $J_{i}(z)=G_{i}(z) / K_{i}(z), 1 \leqq i \leqq$ 3. Then $J_{i}(z)$ is non zero in $D^{*}$ and $J_{1}\left(z_{1}\right) J_{2}\left(z_{2}\right) J_{3}\left(z_{1} z_{2}\right)=1$ for all $z_{1}, z_{2}$ in $D^{*}$. Substituting $z_{2}=1$, it can be seen that $J_{1}\left(z_{1}\right) J_{3}\left(z_{1}\right)=1$ for all $z_{1} \in D^{*}$. Similarly we get that $J_{2}\left(z_{2}\right) J_{3}\left(z_{2}\right)=1$ for all $z_{2} \in D^{*}$. Hence $J_{3}\left(z_{1}\right) J_{3}\left(z_{2}\right)=J_{3}\left(z_{1} z_{2}\right)$ for all 
$z_{1}, z_{2} \in D^{*}$. Further $J_{3}$ is continuous in $D^{*}$. But the only continuous solution of this equation are functions of the type $\Pi_{j=1}^{K} z_{i}^{c_{i}}$ where $c_{j}$ are constants by results of Aczel (1966), p. 215. Hence $G_{3}(z)=K_{3}(z) \Pi_{j=1}^{K} z_{j}^{c^{i}}$ for all $z \in D^{*}$. Splitting the product $\Pi_{j=1}^{K} z_{j}^{c_{1}}$ into two parts consisting of the positive and negative $c_{j}$ 's respectively, it can be seen that

$$
\prod_{j=1}^{K} z_{i}^{b_{i}} G_{3}(z)=\prod_{i=1}^{K} z_{j}^{d_{i}} K_{3}(z), b_{j} \geqq 0, d_{i} \geqq 0
$$

for all $z \in D^{*}$. Since both sides of $\left(^{*}\right)$ are analytic in $D=\left\{\left|z_{j}\right|<1,1 \leqq j \leqq K\right\}$ and they agree on a subset $D^{*}$ which has a limit point in $D$, they agree on $D$ by analytic continuation. In other words $G_{3}(\xi)=K_{3}(\xi) J_{3}(\xi)$ for every $\xi$ which is of the form

$$
1-\sum_{j=1}^{K}\left(1-z_{j}\right) \chi_{A_{j}}(t), 0 \leqq z_{j} \leqq 1,1 \leqq j \leqq K,
$$

where $J_{3}(\xi)=\Pi_{j=1}^{K} z_{i}^{c_{i}}, c_{j}$ real.

$\chi_{A}$ is the indicator function of set $A$. Define $J_{3}(\xi)=\lim J_{3}\left(\xi_{n}\right)$ for any $\xi \in V$. This is possible since every $\xi \in V$ can be uniformly approximated by an increasing sequence of simple functions of the above type. Note that $G_{3}$ and $K_{3}$ are continuous. Hence it follows that $G_{3}(\xi)=K_{3}(\xi) J_{3}(\xi)$ for all $\xi \in V$ where $J_{3}(\xi)$ is the p.g.fl of a degenerate point process by Westcott (1972). But the p.g.fl uniquely determines the point process by Proposition 1 of Vere-Jones (1968). Hence $N_{3}$ and $R_{3}$ differ by a degenerate point process. Similar argument shows that $N_{1}, R_{1}$ and $N_{2}, R_{2}$ differ by degenerate point processes. But the structure of the bivariate process $\left(M_{1}, M_{2}\right)$ shows that we cannot add a degenerate process to one component without subtracting it from another. Hence $N_{1}, N_{2}$ and $N_{3}$ are unique to the process $\left(M_{1}, M_{2}\right)$.

\section{Remarks}

Milne (1970) considers a system in which each point of a stationary Poisson process is subjected to a random displacement, the displacements being independent and identically distributed. He proved that the displacement distribution is identifiable if a complete input-output record is given but not the linkage between the two. Theorem 2.1 is similar to his result except that no assumptions are made about the distributions of the processes involved.

\section{Acknowledgement}

The author thanks the referee for his pertinent comments and suggestions on earlier versions of this note. 


\section{References}

J. Aczel (1966), Lectures on functional equations and their applications (Academic Press, 1966).

T. E. Harris (1963), The theory of branching processes (Springer-Verlag, 1963).

R. K. Milne (1970), 'Identifiability for random translations of Poisson processes', Z. Wahrscheinlichkeitstheorie und Verw. Gebiete 15, 195-201.

D. Vere-Jones (1968), 'Some applications of probability generating functionals to the study of input-output streams', J. Roy. Statistic Soc. Ser. B 30, 321-333.

M. Westcott (1972), 'The probability generating functional', J. Austral. Math. Soc. 14, 448-466.

\section{Department of Mathematics}

Indian Institute of Technology

Kanpur 208016, India.

Present address:

Centre de recherches mathématiques

Université de Montréal

Case Postale 6128

Montréal H3C 3J7

Canada. 